\title{
Retroperitoneal fibrosis and constrictive pericarditis-IgG4 related diseases: A case report
}

\author{
BRANKICA TERZIC ${ }^{1}$, MARIJAN SPASIC ${ }^{2}$, PREDRAG DJURIC ${ }^{2}$, \\ VLADIMIR VASILJEVIC ${ }^{3}$, SLAVICA RADJEN ${ }^{4}$ and MIRJANA MIJUSKOVIC ${ }^{1}$ \\ ${ }^{1}$ Nephrology and ${ }^{2}$ Cardiology Clinics; Institutes of ${ }^{3}$ Pathology and ${ }^{4}$ Hygiene, \\ Military Medical Academy, 11000 Belgrade, Serbia
}

Received June 16, 2016; Accepted January 20, 2017

DOI: $10.3892 / \mathrm{etm} .2017 .4425$

\begin{abstract}
Retroperitoneal fibrosis (RPF) is a rare disease characterized by infiltration of inflammatory cells and deposition of thickened fibrous tissues. The present study presents the case of a 53-year-old patient treated for generalized weakness and fatigue for 1 year prior to hospitalization. A cardiac ultrasound revealed pericardial effusion that required pericardiocentesis, during which $1,400 \mathrm{ml}$ serous fluid with the characteristics of an exudate was aspirated. A pericardiectomy was performed due to persistent effusion and histological examination indicated pericardial fibrosis. A thoracic-abdominal computed tomography scan revealed the presence of retroperitoneal fibrosis. The patient was treated with corticosteroids and azathioprine. Follow-up examinations showed a significant reduction in the amount of abdominal fibrous tissue and no increase in pericardial effusion 1 year following the end of treatment. The patient continues to have regular follow-up control examinations with a cardiologist and nephrologist.
\end{abstract}

\section{Introduction}

Retroperitoneal fibrosis (RPF) is characterized by the presence of fibro-inflammatory tissue in the retroperitoneum that covers neighboring structures (1). This disease is rare, with an incidence rate of 1 in 200,000 (2). RPF typically presents in individuals 40-60 years of age and is 2-3 times more common in males than females $(3,4)$.

There are two forms of RPF. The first, idiopathic RPF, makes up $\sim 70 \%$ of cases (5) and may be linked with other immunoglobulin (Ig) G4 related diseases (6). The other, more rare form of RPF is secondary RPF, which is

Correspondence to: Dr Brankica Terzic, Nephrology Clinic, Military Medical Academy, 17 Crnotravska St, 11000 Belgrade, Serbia

E-mail: brankica.terzic@gmail.com

Key words: retroperitoneal fibrosis, constrictive pericarditis, immunoglobulin G4 related disease associated with malignancy, infection, drug use and other primary causes $(1,4,7)$.

RPF typically manifests clinically with back pain, generalized weakness and anemia, and occasionally with renal failure and hydronephrosis $(4,8,9)$. Analysis of blood samples from patients with RPF typically shows increased erythrocyte sedimentation rate and C-reactive proteins, and sometimes anemia and increased levels of urea and creatinine $(4,10)$. The results of a previous study found that IgG4 was increased in $60-70 \%$ patients with IgG4 related diseases (11). Constrictive pericarditis and pericardial fibrosis combined with retroperitoneal fibrosis is extremely rare and has been depicted only in a few cases in medical literature (12-14). An elevated level of immunoglobulin (Ig) G4 is characteristic of these diseases and so they are categorized into the hyper IgG4 related disease group.

\section{Case report}

A 53-year-old male patient was admitted to the Cardiology Clinic (Military Medical Academy, Belgrade, Serbia) in January 2009 due to generalized weakness and fatigue lasting 1 year. An initial physical examination revealed no pathological signs; heart sounds were unremarkable and blood pressure was 140/80 mmHg. Echocardiography revealed pericardial effusion $1.7 \mathrm{~cm}$ in front of the right ventricle and $2.5 \mathrm{~cm}$ behind the left ventricle. A radiograph of the chest showed signs of an enlarged heart and pericardial effusion. An electrocardiogram disclosed a sinus rhythm of 64 per min and low voltage QRS without T-wave abnormalities. Complete blood cell count and biochemical screening was normal. Erythrocyte sedimentation rate (ESR) was elevated up to $57 \mathrm{~mm} / \mathrm{h}$ (normal value $<20 \mathrm{~mm} / \mathrm{h}$ ) and levels of C-reactive protein (CRP) were elevated up to $56.7 \mathrm{mg} / \mathrm{l}$ (normal value, 1.0 to $3.0 \mathrm{mg} / \mathrm{l}$ ). The titer of Coxsackie B4 and B5 virus was $>1: 1,024$ (normal value 1:16), whereas levels of serum protein electrophoresis, immunoglobulins, C3, C4, anti-nuclear antibody and thyroid hormones were within normal ranges. Pericardiocentesis was performed twice and >1,400 $\mathrm{ml}$ serous fluid, with the characteristics of an exudate, was aspirated. Malignant cells and mycobacterium were not observed. The patient was treated with Ceftriaxon at a dose of $1 \mathrm{~g}$ per day, for 10 days. The patient was administered Ibuprofen at a dosage of $800 \mathrm{mg}$ per day for the first 7 days, and 

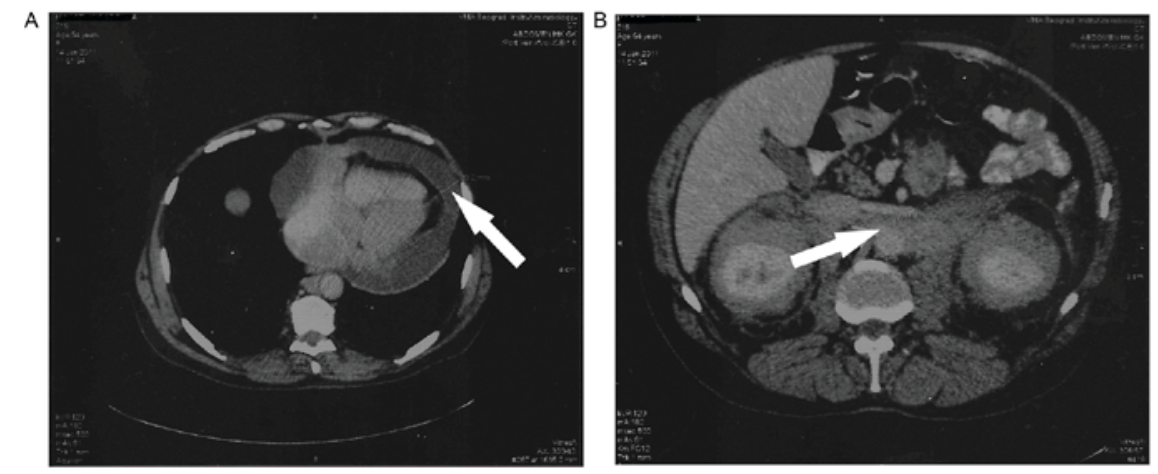

Figure 1. (A) Computed tomography scan images of the pericardial effusion, up to $20 \mathrm{~mm}$ thick. (B) Perineal and para-aortic changes characteristics for retroperitoneal fibrosis. Arrow indicates fibrous tissue.
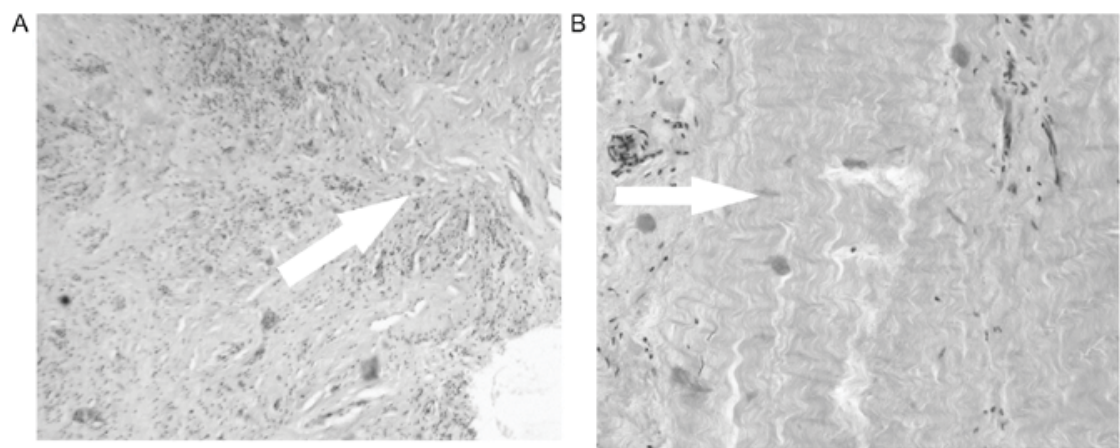

Figure 2. Initial scaring following chronic inflammation of the analyzed tissue. (A) Granulomatous tissue with initial scarring. (B) Thick fibrous tissue of the pericardium. Arrow indicates fibrous tissue.

$400 \mathrm{mg}$ per day for the following two weeks which improved the general condition and the patient was discharged after 30 days. The patient was hospitalized again 3 months later with the same symptoms; an effusion was identified $1.2 \mathrm{~cm}$ in front of the right atrium and $1.4 \mathrm{~cm}$ behind the left ventricle. The patient began taking prednisone (Galenika, a.d., Beograd, Serbia) at a dose of $40 \mathrm{mg}(0.5 \mathrm{mg} / \mathrm{kg})$ per day for 1 week, $30 \mathrm{mg}$ for 1 week, $20 \mathrm{mg}$ for 2 weeks and $10 \mathrm{mg}$ for the next 2 months. Following 3 months treatment, the corticosteroids were discontinued. A follow-up control examination registered again the pericardial effusion $1.9 \mathrm{~cm}$ behind the left ventricle and $1.2 \mathrm{~cm}$ in front of the right ventricle. Following a complete blood cell count and biochemical screening, laboratory results were within the reference ranges, with the exception of $\mathrm{C}$-reactive protein (CRP) level, which was $29.9 \mathrm{mg} / \mathrm{l}$ (normal value, 1.0-3.0 mg/l) and erythrocyte sedimentation rate (ESR) $46 \mathrm{~mm} / \mathrm{h}$ (normal value, up to $20 \mathrm{~mm} / \mathrm{h}$ ) At 1 year post-treatment, a computed tomography (CT) scan of the chest and abdomen revealed a large pericardial effusion up to $2.0 \mathrm{~cm}$ thick (Fig. 1A) and in the abdomen, perirenal and para-aortic hypodense zones were also identified, which may be the result of pronounced retroperitoneal fibrosis (Fig. 1B).

Pericardiectomy was performed due to constrictive pericarditis. During surgery, the pericardium was found to be $>1 \mathrm{~cm}$ thick with calcifications, with a tough consistency and thickness of mucosa which was $>5 \mathrm{~mm}$. These macroscopic features suggested pericardial fibrosis. Histopathology result, obtained from four samples confirmed that diagnosis (Fig. 2) and the patient was treated with $15 \mathrm{mg} /$ day prednisone.
At 3 months post-surgery, the patient was examined by a nephrologist. A kidney echo tomography identified hydronephrosis stage I/II with no abnormalities. The dosage of prednisone was increased to $40 \mathrm{mg}$; however, 1 month later diabetes mellitus iatrogenic was diagnosed. Therefore, prednisone was reduced to $10 \mathrm{mg} /$ day and the patient was treated with azathioprine (PharmaSwiss AG; Valeant Phrarmaceuticals International, Inc., Laval, Canada) at a dosage of $100 \mathrm{mg}$ per day. A control follow-up examination was performed 1 month later and showed improvement in the renal ultrasound image in the terms of withdrawal of the hydronephrosis on the left kidney. At 6 months after dual immunosuppressive therapy, another thoracic-abdominal CT scan was performed. This revealed a thickened pericardium and encapsulated effusion with a diameter of $10 \mathrm{~mm}$ (Fig. 3A). The retroperitoneal fibrosis was still present in the abdomen, but with less intensity than prior to the introduction of immunosuppressive therapy (Fig. 3B).

IgG4 serum level was first measured at this point and was found to be elevated to $1.63 \mathrm{~g} / \mathrm{l}$ (normal value, $\leq 1.4 \mathrm{~g} / \mathrm{l}$ ), whereas levels of $\operatorname{IgG}$ and other Igs were within reference ranges.

The patient did not present with any pain and the echocardiography report was unchanged from previous reports. Treatment for RPF and pericardial fibrosis was continued with Imuran $(75 \mathrm{mg} /$ day; PharmaSwiss, AG; Valeant Pharmaceuticals International, Inc.) and prednisone (10 mg/day). One year later, the Imuran dosage was reduced to $50 \mathrm{mg} / \mathrm{day}$, follow-up assessments were completed for the current patient every 2 months for the first 2 years and then every 4 months thereafter. This immunosuppressive dosage 

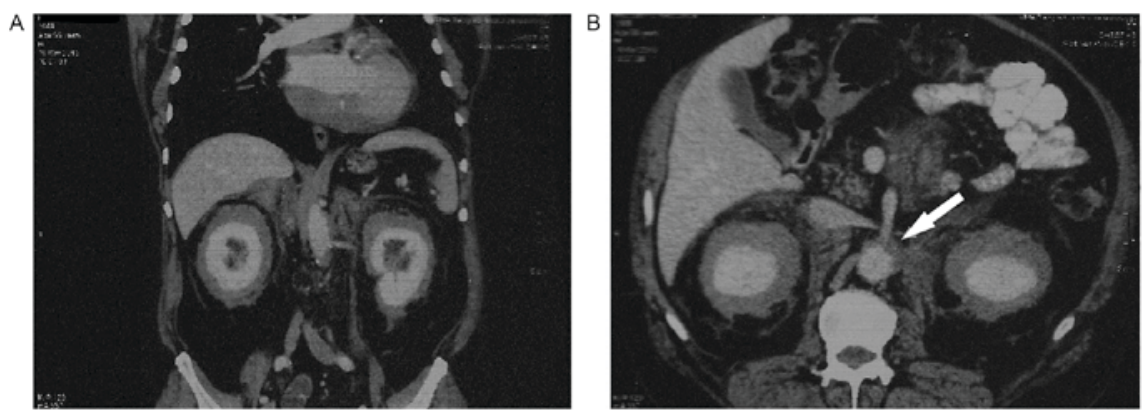

Figure 3. (A) Computed scan images 6 months after therapy (encapsulated pericardial effusion). (B) The intensity of retroperitoneal fibrosis was lower than before therapy. Arrow indicates fibrous tissue.
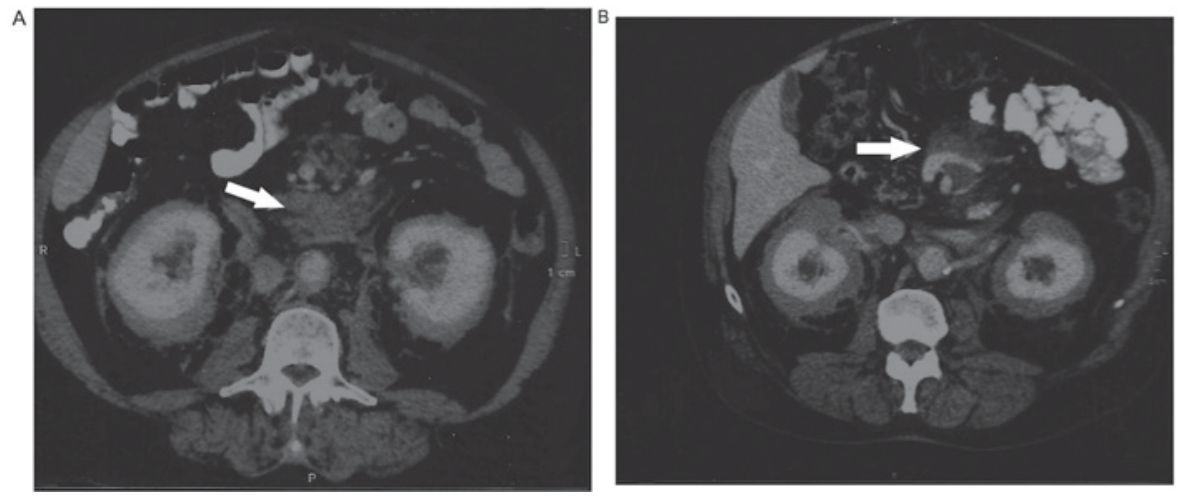

Figure 4. (A and B) Computed tomography scan demonstrating that the fibrous tissue had regressed after 6 months of treatment. Arrow indicates fibrous tissue.

was maintained for 1 year, giving a total of 3 years following the introduction of dual immunosuppressive therapy. Another thoracic-abdominal CT scan was performed and the image revealed that fibrosis had regressed compared with the previous scans (Fig. 4). Imuran treatment was discontinued, as was pronisone treatment 3 months later. Laboratory and ultrasound follow-up control examinations were continued, including a complete blood cell count, biochemical screening, serum protein electrophoresis and assessments of immunilobulin, CRP and ESR. In total, the patient was treated for nearly 4 years. All laboratory results were within the reference ranges. At 1 year after treatment was discontinued, the patient had no subjective symptoms and an ultrasound of the kidneys and heart showed no change.

\section{Discussion}

RPF is an extremely rare disease that affects males more often than females, with a ratio of $\sim 3: 1$. RPF typically presents with inflammatory and fibrous tissue in the retroperitoneum that compresses the organs (1-3). Laboratory tests exhibit typically unremarkable remarks, aside from an elevated ESR and CRP levels in the majority of patients, although acute kidney failure also occurs in some cases (4). RPF is typically diagnosed based on radiographic images (CT scans) as symptoms are diverse and range from mild back pain and generalized weakness to the development of obstructive acute kidney failure $(4,5)$. In a small number of cases, RPF is combined with pericardial fibrosis. Previous studies of RPF have revealed the presence of Ig in these diseases, categorizing them as IgG4 related diseases $(12,14,15)$.
In the present study, the disease first manifested with refractory pericardial effusion, which was temporarily suppressed by treatment with prednisone. At 18 months following the first echocardiography, pericardiocentesis was performed. A subsequent histopathology report revealed pericardial fibrosis and a thoracic-abdominal CT scan identified massive changes characteristic of retroperitoneal fibrosis, confirming a diagnosis of RPF with pericardial fibrosis $(7,9)$. The patient was started on $0.5 \mathrm{mg} / \mathrm{kg}$ prednisone and $100 \mathrm{mg} /$ day azathioprine. Unlike the majority of patients, the patient in the current study presented with a very refractory form of disease, requiring continued dosage reduction, and the total duration of therapy was 36 months, while the average duration of treatment was 24 months (10). IgG4 was detected for the first time 1 year following the commencement of therapy and was found to be slightly elevated. The results confirm that finding these extremely rare diseases are IgG4 related $(11,12)$.

IgG4 related diseases are very rare and prognosis depends on clinical manifestation and the degree to which organs are affected. Diagnoses are typically made via imaging studies (CT scan) and patients are typically treated with prednisone, or a combination of prednisone and azathioprine or mycophenolic acid. The duration of treatment depends on how quickly fibrous tissue is suppressed and ranges from a few months to a few years.

In conclusion, the results of the current study indicated that for middle-aged patients who present with generalized weakness, back pain and elevated ESR and CRP levels, retroperitoneal fibrosis and pericardial fibrosis should be considered as a possible diagnosis. 


\section{References}

1. Vaglio A, Salvarani C and Buzio C: Retroperitoneal fibrosis. Lancet 367: 241-251, 2006.

2. Khan AN: Retroperitoneal Fibrosis, eMedicine, Feb 2008.

3. Vaglio A, Palmisano A, Alberici F, Maggiore U, Ferretti S, Cobelli R, Ferrozzi F, Corradi D, Salvarani C and Buzio C: Prednisone versus tamoxifen in patients with idiopathic retroperitoneal fibrosis: An open-label randomised controlled trial. Lancet 378: 338-346, 2011.

4. van Bommel EF, Jansen I, Hendriksz TR and Aarnoudse AL: Idiopathic retroperitoneal fibrosis: Prospective evaluation of incidence and clinicoradiologic presentation. Medicine (Baltimore) 88: 193-201, 2009.

5. Cronin CG, Lohan DG, Blake MA, Roche C, McCarthy P and Murphy JM: Retroperitoneal fibrosis: A review of clinical features and imaging findings. AJR Am J Roentgenol 191: 423-431, 2008

6. Zen Y, Sawazaki A, Miyayama S, Notsumata K, Tanaka N and Nakanuma Y: A case of retroperitoneal and mediastinal fibrosis exhibiting elevated levels of IgG4 in the absence of sclerosing pancreatitis (autoimmune pancreatitis). Hum Pathol 37: 239-243, 2006.

7. Goenka AH, Shah SN and Remer EM: Imaging of the retroperitoneum. Radiol Clin North Am 50: 333-355.vii, 2012.

8. Gilkeson GS and Allen NB: Retroperitoneal fibrosis. A true connective tissue disease. Rheum Dis Clin North Am 22: 23-38, 1996.

9. van Bommel EF, van Spengler J, van der Hoven B and Kramer P Retroperitoneal fibrosis: Report of 12 cases and a review of the literature. Neth J Med 39: 338-345, 1991.

10. Magrey MN, Husni ME, Kushner I and Calabrese LH: Do acute-phase reactants predict response to glucocorticoid therapy in retroperitoneal fibrosis? Arthritis Rheum 61: 674-679, 2009.

11. Stone JH, Zen Y and Deshpande V: IgG4-related disease. N Engl J Med 366: 539-551, 2012

12. Khosroshahi A and Stone JH: A clinical overview of IgG4-related systemic disease. Curr Opin Rheumatol 23: 57-66, 2011.

13. Sugimoto T, Morita Y, Isshiki K, Yamamoto T, Uzu T, Kashiwagi A, Horie M and Asai T: Constrictive pericarditis as an emerging manifestation of hyper-IgG4 disease. Int J Cardiol 130: e100-e101, 2008.

14. Takikita-Suzuki M, Ishida M and Okabe H: Re-evaluation of IgG4 in systemic fibroinflammatory disease with intracardiac involvement. Hum Pathol 41: 458-459, 2010.

15. Sakamoto A, Nagai R, Saito K, Imai Y, Takahashi M, Hosoya Y, Takeda N, Hirano K, Koike K, Enomoto Y, et al: Idiopathic retroperitoneal fibrosis, inflammatory aortic aneurysm, and inflammatory pericarditis-retrospective analysis of 11 case histories. Int J Cardiol 59: 139-146, 2012. 